\title{
The Iterated Sinh Transformation
}

\author{
David Elliott, \\ School of Mathematics and Physics, \\ University of Tasmania, \\ Private Bag 37, \\ Hobart, \\ Tasmania, 7001, \\ Australia. \\ E-mail: elliott@hilbert.maths.utas.edu.au \\ Peter R. Johnston * \\ School of Biomolecular and Physical Sciences, \\ Griffith University, \\ Nathan, \\ Queensland, 4111, \\ Australia. \\ Phone: 61-7-3735-7748, \\ Fax: 61-7-3735-7656, \\ E-mail: P.Johnston@ @riffith.edu.au \\ September 14, 2007
}

*Author to whom correspondence should be addressed. 


\begin{abstract}
A sinh transformation has recently been proposed to improve the numerical accuracy of evaluating nearly singular integrals using Gauss-Legendre quadrature. It was shown that the transformation could improve the accuracy of evaluating such integrals, which arise in the boundary element method, by several orders of magnitude. Here, this transformation is extended in an iterative fashion to allow the accurate evaluation of similar types of integrals which have more spiked integrands.

Results show that one iteration of this sinh transformation is preferred for nearly weakly singular integrals, whereas two iterations lead to several orders of magnitude improvement in the evaluation of nearly strongly singular integrals. The same observation applies when considering integrals of derivatives of the two-dimensional boundary element kernel. However, for these integrals, more iterations are required as the distance from the source point to the boundary element decreases.
\end{abstract}

Keywords: non-linear co-ordinate transformation; boundary element method; nearly singular integrals; numerical integration; sinh function 


\section{Introduction}

Implementation of the boundary element method requires the accurate numerical evaluation of integrals of the form

$$
I=\int_{-1}^{1} g(x) f\left(\left(x-a_{0}\right)^{2}+b_{0}^{2}\right) d x
$$

where it is assumed that $-1<a_{0}<1$ and $0<b_{0} \ll 1$. The function $f$ is assumed to have some singularity (i.e. pole or branch point) when its argument is zero, that is, $f$ has singularities at points $z_{0}$ and $\overline{z_{0}}$ where $z_{0}=a_{0}+\mathrm{i} b_{0}$. Although the integral $I$ is not singular, since we are assuming that $0<b_{0}<<1$, it is said to be a "nearly singular" integral. The function $g$ is assumed to be "well behaved". Here, three particular forms of the function $f$ will be considered; say

$$
f_{1}(r)=\ln r, \quad f_{2}(r)=\frac{1}{r}, \quad f_{3}(r)=\frac{1}{r^{\lambda}}
$$

where $r^{2}=\left(x-a_{0}\right)^{2}+b_{0}^{2}$ and $\lambda>0$ is not necessarily an integer.

Numerical evaluation of integrals of the above forms has received considerable attention over many years $[1,2,3,4,5,6,7]$. These studies are all based on Gauss-Legendre quadrature but either use a polynomial transformation of the Gauss-Legendre integration points or split the interval of integration at the "nearly singular point", $a_{0}$, and apply transformations in each subinterval.

Recently a transformation, based on the sinh function, was introduced to evaluate nearly singular integrals [8]. One advantage of this new transformation is that it automatically incorporates the position of the nearly singular point $a_{0}$ into the transformation, as well as the distance $b_{0}$ from the source point to the element. It was also demonstrated that the sinh transformation provided a significant improvement over the untransformed use of Gauss-Legendre quadrature and could be applied to a wide variety of problems.

It was also mentioned in [8] that the sinh transformation performed better on "nearly weakly singular integrals" than it did with "nearly strongly singular integrals". In the context of this paper, a "nearly weakly/strongly singular integral" is defined as an integral which would in fact be 
weakly/strongly singular if the source point was on the element over which the integration was being performed. These integrals are characterised by integrands which are continuous over the range of integration, but which exhibit a high narrow spike around the nearly singular point $a_{0}$.

With the above considerations in mind, this paper introduces an iterated sinh transformation which is applicable to nearly strongly singular integrals. It is shown that the iterated sinh transformation can further reduce relative errors in the numerical evaluation of nearly strongly singular integrals by several orders of magnitude.

In the next section the iterated sinh transformation is introduced and several of its properties discussed. Section 3 then considers several examples where zero, one and two iterations of the sinh transformation are used, as well as two existing transformations, and compares their relative errors. Finally, Section 4 makes some recommendations as to the use of the iterated sinh transformation.

\section{The Iterated Sinh Transformation}

For the integral $I$ of (1) the sinh transformation has been defined previously [8] as

$$
x=a_{0}+b_{0} \sinh \left(\mu_{0} u-\eta_{0}\right) .
$$

It then follows that

$$
d x=b_{0} \mu_{0} \cosh \left(\mu_{0} u-\eta_{0}\right) d u
$$

and

$$
\left(x-a_{0}\right)^{2}+b_{0}^{2}=b_{0}^{2} \cosh ^{2}\left(\mu_{0} u-\eta_{0}\right) .
$$

Upon choosing

$$
\mu_{0}=\frac{1}{2}\left\{\operatorname{arcsinh}\left(\frac{1+a_{0}}{b_{0}}\right)+\operatorname{arcsinh}\left(\frac{1-a_{0}}{b_{0}}\right)\right\}
$$

and

$$
\eta_{0}=\frac{1}{2}\left\{\operatorname{arcsinh}\left(\frac{1+a_{0}}{b_{0}}\right)-\operatorname{arcsinh}\left(\frac{1-a_{0}}{b_{0}}\right)\right\}
$$


it turns out that $-1 \leq x \leq 1$ maps into $-1 \leq u \leq 1$ so that, from (1),

$$
I=b_{0} \mu_{0} \int_{-1}^{1} g(x(u)) \cosh \left(\mu_{0} u-\eta_{0}\right) f\left(b_{0}^{2} \cosh ^{2}\left(\mu_{0} u-\eta_{0}\right)\right) d u
$$

For appropriate forms of the function $f$ the integrand will have singularities where

$$
\cosh \left(\mu_{0} u-\eta_{0}\right)=0
$$

In the complex plane there are an infinite number of solutions of this equation, but the nearest singularities to the interval $(-1,1)$ are at $z_{1}$ and $\overline{z_{1}}$ say, where

$$
z_{1}:=a_{1}+\mathrm{i} b_{1}
$$

with

$$
a_{1}=\frac{\eta_{0}}{\mu_{0}} \quad \text { and } \quad b_{1}=\frac{\pi}{2 \mu_{0}}
$$

It has been shown [9, Theorem 3.4] that the points $z_{1}$ and $\overline{z_{1}}$ are "further away" from the interval $(-1,1)$ than are the original points $z_{0}$ and $\overline{z_{0}}$ so that applying the same $n$-point Gauss-Legendre quadrature rule is likely to lead to smaller truncation errors than for the original integral.

It would therefore appear reasonable to apply this approach again and define an iterated sinh transformation by letting

$$
u=a_{1}+b_{1} \sinh \left(\mu_{1} v-\eta_{1}\right),
$$

where

$$
\mu_{1}=\frac{1}{2}\left\{\operatorname{arcsinh}\left(\frac{1+a_{1}}{b_{1}}\right)+\operatorname{arcsinh}\left(\frac{1-a_{1}}{b_{1}}\right)\right\}
$$

and

$$
\eta_{1}=\frac{1}{2}\left\{\operatorname{arcsinh}\left(\frac{1+a_{1}}{b_{1}}\right)-\operatorname{arcsinh}\left(\frac{1-a_{1}}{b_{1}}\right)\right\} .
$$

The Jacobian of this transformation is now

$$
d u=b_{1} \mu_{1} \cosh \left(\mu_{1} v-\eta_{1}\right) d v
$$


and, from (8), the integral $I$ becomes

$I=\frac{\pi b_{0} \mu_{1}}{2} \int_{-1}^{1} g(x(u(v))) \cosh \left(\mu_{1} v-\eta_{1}\right) \cosh \left(\pi \sinh \left(\mu_{1} v-\eta_{1}\right) / 2\right) f\left(b_{0}^{2} \cosh ^{2}\left(\pi \sinh \left(\mu_{1} v-\eta_{1}\right) / 2\right) d v\right.$,

since, from (11), $\mu_{0} a_{1}-\eta_{0}=0$ and $\mu_{0} b_{1}=\pi / 2$. The singularities of appropriate forms of the function $f$ will now be even further removed from the interval of integration $(-1,1)$ than before, so that $n$-point Gauss-Legendre quadrature should be even better.

The idea behind a transformation technique for evaluating nearly singular integrals is to shift the integration points to regions where the integrand exhibits a spike. As discussed previously in [8], the sinh transformation automatically accounts for the nearly singular point and its distance from the interval of integration. Figure 1 shows the effect the transformations (3) and (12) have on the original Gauss-Legendre nodes points (Iteration 0) with $a_{0}=0.25$ and $b_{0}=0.01$, for 20 integration points. Such a transformation would be applicable when the function $f$ has a near singularity at $x=0.25$. Notice that the original Gauss-Legendre quadrature points are clustered towards the ends of the interval of integration and there are few near the point of interest at $x=0.25$. It can be seen that the first iteration (Iteration 1) clusters the integration points around the nearly singular point. A second iteration of the transformation clusters the integration points even more strongly around $x=0.25$. Hence, for an integral which is nearly strongly singular, it would be expected that the second iteration of the sinh transformation would more accurately evaluate the integral but, as we shall see in Section 3 , this is not always the case.

\section{Results and Discussion}

To demonstrate the accuracy of the above transformations, several example integrals will be evaluated. The numerical values obtained will be compared to exact values in terms of the relative error defined 
by

$$
\text { Relative Error }=\frac{\left|I_{\text {numerical }}-I_{\text {exact }}\right|}{\left|I_{\text {exact }}\right|}
$$

where $I_{\text {numerical }}$ is the value obtained for the integral using the indicated numerical method and $I_{\text {exact }}$ is the exact value of the integral. The integrals in subsections $\S 3.1$ and $\S 3.2$ can be obtained analytically using the tables of integrals $[10,11]$. Their values are given in Table 1. "Exact" values for the examples in subsection $\S 3.3$ were obtained numerically using the quadl routine in MATLAB to a tolerance of $10^{-15}$.

Results are also compared with the transformation methods of Telles and Oliveira [2] and of Ma and Kamiya [7]. Comparisons are made with the Telles and Oliveira transformation only for the integrals with $\ln r, r^{-1}, r^{-2}$ and $r^{-3}$ type kernels as these were the integrals for which the original transformation was designed. Also, comparisons are made only for $b_{0}=0.1$ and $b_{0}=0.01$ since parameter values used in the transformation are available only for these distances. In comparisons with the transformation of Ma and Kamiya, it should be noted that this transformation splits the interval of integration into two subintervals at the point $a_{0}$, so in order to achieve a fair comparison in terms of computational load, only half the indicated number of nodes are used in each subinterval, giving the same total number of function evaluations for all methods. However, in Table 2, when 15 and 25 integration points are used for the Telles and Oliveira and iterated sinh transformations, 8 and 13 integration points, respectively, were used on each subinterval in the Ma and Kamiya transformation so that in both cases there is an additional function evaluation.

\subsection{Nearly Weakly Singular Integrals}

Consider the numerical evaluation of the integrals

$$
I_{1}=\int_{-1}^{1}\left(1-x^{2}\right) \ln \left(\left(x-\frac{1}{4}\right)^{2}+b_{0}^{2}\right) d x
$$


and

$$
I_{2}=\int_{-1}^{1} \frac{1-x^{2}}{\sqrt{\left(x-\frac{1}{4}\right)^{2}+b_{0}^{2}}} d x
$$

The integral $I_{1}$ is nearly weakly singular in the sense of the definition given in Section 1 . However, although the integral $I_{2}$ with $b_{0}=0$ is actually a Hadamard finite-part integral, in this context it will be considered to be nearly weakly singular in the sense that the transformation (3) removes the nearly singular, or spiked, behaviour of the integrand.

The integral $I_{1}$ represents an integral over a straight boundary element with the logarithmic kernel and a basis function $g(x)=1-x^{2}$. The nearly singular point is at $x=\frac{1}{4}$ and the distance from the source point to the element is given by $b_{0}$. The integral $I_{2}$ also represents integration over a straight element, this time with a $\frac{1}{r}$ type integrand and the same basis function. This integral has been considered previously in [8].

Table 2 shows the relative errors for numerical evaluation of the integral $I_{1}$ with the nearly singular point at $x=\frac{1}{4}$ for various values of $b_{0}$ and various numbers of integration points, $n$. It can be seen from the table that in all cases one iteration of the transformation leads to a considerable improvement of the accuracy of the numerical calculation over using straightforward Gauss-Legendre quadrature (no iterations). Also, the transformations of Telles and Oliveira and Ma and Kamiya are an improvement over standard Gauss-Legendre quadrature, but are not as accurate as one iteration of the sinh transformation. For the largest $b_{0}$ value considered, the Telles and Oliveira transformation is more accurate than the Ma and Kamiya transformation.

Note that in most cases a second iteration of the transformation yields less accurate results, especially at small $b_{0}$ values. One possible explanation is that the second iteration unnecessarily pushes too many integration points towards the nearly singular point, resulting in insufficient evaluation of the integrand away from this point. From another point of view, one iteration of the sinh transformation 
results in the integral

$$
I_{1}=2 b_{0} \mu_{0} \int_{-1}^{1}\left(1-x^{2}(u)\right) \cosh \left(\mu_{0} u-\eta_{0}\right) \ln \left(b_{0} \cosh \left(\mu_{0} u-\eta_{0}\right)\right) d u
$$

where $\mu_{0}$ and $\eta_{0}$ are given by (6) and (7), respectively, with $a_{0}=\frac{1}{4}$. The integral now contains an integrand which has had its spike removed. In this case, one iteration of the sinh transformation is sufficient.

Table 3 shows similar results for the integral $I_{2}$. Again, two iterations of the sinh transformation generally lead to inferior results. The reason here is that one iteration of the sinh transformation results in the integral

$$
I_{2}=\mu_{0} \int_{-1}^{1}\left(1-x^{2}(u)\right) d u
$$

for which the integrand contains no spike. Again, one iteration of the sinh transformation is sufficient.

\subsection{Nearly Strongly Singular Integrals}

Consider the numerical evaluation of the integrals

$$
\begin{aligned}
& I_{3}=\int_{-1}^{1} \frac{1-x^{2}}{\left(x-\frac{1}{4}\right)^{2}+b_{0}^{2}} d x \\
& I_{4}=\int_{-1}^{1} \frac{1-x^{2}}{\left(\left(x-\frac{1}{4}\right)^{2}+b_{0}^{2}\right)^{\frac{3}{2}}} d x,
\end{aligned}
$$

and

$$
I_{5}=\int_{-1}^{1} \frac{1-x^{2}}{\left(\left(x-\frac{1}{4}\right)^{2}+b_{0}^{2}\right)^{2}} d x
$$

These examples are more difficult to evaluate accurately numerically than those of the previous section in that the near singularity cannot be removed by the transformation. Also, the spike in the integrand is considerably narrower and higher than in the previous examples, suggesting that more integration points should be clustered around the nearly singular point. 
Table 4 shows the relative errors for the numerical evaluation of the integral $I_{3}$ with the nearly singular point at $x=\frac{1}{4}$ and various values of $b_{0}$. This table shows that whilst one iteration of the sinh transformation remarkably improves the numerical evaluation of the integral (by several orders of magnitude in many cases), a second iteration improves the numerical evaluation again (also by several orders of magnitude). The improvement is often more dramatic at smaller values of $b_{0}$. Also at small $b_{0}$ values the transformation of Ma and Kamiya is generally superior to one iteration of the sinh transformation.

Similar results are shown in Tables 5 and 6 for the numerical evaluation of the integrals $I_{4}$ and $I_{5}$, respectively. In both cases applications of one and two iterations of the sinh transformation lead to similar levels of improvement to that shown for the numerical evaluation of the integral $I_{3}$.

\subsection{Boundary Element Derivative Examples}

Finally, consider the integrals of derivatives of the logarithmic boundary element kernel

$$
I_{6}=\int_{\Gamma_{e}} \frac{g}{r} \frac{\partial r}{\partial x} d \Gamma
$$

and

$$
I_{7}=\int_{\Gamma_{e}} g \frac{\partial}{\partial x}\left(\frac{1}{r} \frac{\partial r}{\partial x}\right) d \Gamma
$$

where $\Gamma_{e}$ is the arc of a unit circle subtending an angle of $10^{\circ}$ and $r^{2}=\left(x-x_{0}\right)^{2}+\left(y-y_{0}\right)^{2}$ with $\left(x_{0}, y_{0}\right)$ being the coordinate of the source point and $(x, y)$ the coordinates of general points along the element $\Gamma_{e}$. The integrals are transformed so that they are mapped onto the interval $[-1,1]$ in terms of some intrinsic coordinate $\xi$ along the element. After the transformation, $r$ becomes $\sqrt{(\xi-a)^{2}+b^{2}}$ where $a$ is the position of the nearly singular point and $b$ the distance to the element. Using the quadratic basis function $g(\xi)=1-\xi^{2}$, the integrals become (using the ideas described in [8])

$$
I_{6}=\int_{-1}^{1} \frac{\left(1-\xi^{2}\right)(\xi-a)}{(\xi-a)^{2}+b^{2}} \sqrt{4\left(\cos \frac{\pi}{36}-1\right)^{2} \xi^{2}+\sin ^{2} \frac{\pi}{36}} d \xi
$$


and

$$
I_{7}=\int_{-1}^{1} \frac{\left(1-\xi^{2}\right)\left(b^{2}-(\xi-a)^{2}\right)}{\left((\xi-a)^{2}+b^{2}\right)^{2}} \sqrt{4\left(\cos \frac{\pi}{36}-1\right)^{2} \xi^{2}+\sin ^{2} \frac{\pi}{36}} d \xi
$$

Accurate evaluation of these integrals requires a larger number of integration points (even with the transformations) due to the oscillations present in the integrands.

Figure 2 shows the relative errors for the evaluation of the integral $I_{6}$ using 40 integration points for $a=0.2$ and various values of $b$. It can be seen that at the smallest values of $b$, two iterations of the sinh transformation produce an order of magnitude improvement over one iteration. At intermediate values of $b$, say $0.005 \leq b \leq 0.05$, both one and two iterations give similar results, so in this region one iteration would be sufficient. For $b \geq 0.05$, the sinh transformation is not required at all. This example indicates that more transformations should be taken as the value of $b$ decreases. For all but the largest values of $b$, both one and two iterations of the sinh transformation give superior results compared to the transformation of Ma and Kamiya, which in turn is a significant improvement over standard Gauss-Legendre quadrature.

Similar observations can be made for the evaluation of the integral $I_{7}$, as seen in Figure 3. Again, 40 integration points are used with $a=0.2$ and various values of $b$. Here, there is a much larger region of $b$ values for which two iterations are superior to one iteration. A possible explanation is that the integrand in $I_{7}$ has one more oscillation than the integrand in $I_{6}$. For intermediate values of $b$, one iteration of the transformation is clearly sufficient and again for $b \geq 0.05$, no iterations are required.

\section{Conclusions}

Here an extension of a previously published sinh transformation [8], to evaluate nearly singular boundary element integrals, has been introduced. The extension involves using the sinh transformation in an iterative fashion. Examples considered show the effects of zero, one and two iterations and these are compared to other existing transformations. 
The examples considered above show that for nearly weakly singular integrals one iteration of the transformation is superior to no iterations and that two iterations are unwarranted. A possible reason for this is that one iteration applied to a nearly weakly singular integral removes the near singularity, or spike, from the integrand making numerical evaluation of the integral much more accurate.

A second iteration of the transformation becomes superior when considering nearly strongly singular integrals. Here, the additional iteration of the transformation leads to several orders of magnitude improvement in the evaluation of the integrals considered. It could be postulated that the second iteration of the sinh transformation also removes the spike from the integrand. Further, more strongly singular integrals could be evaluated more accurately with further iterations of the sinh transformation.

The final examples show that firstly, more integration points are required when considering derivatives of the logarithmic kernel in the boundary element method, which is due to the oscillatory nature of the integrand, and secondly, that the number of iterations is more closely related to the distance from the source point to the element $b$ than the height of the spike caused by the near singularity. In other words, there is a range of values of $b$ where no iteration is required, another where one iteration would suffice and others where more iterations are required.

Finally, it has been shown previously in [8] that splitting the interval of integration at the nearly singular point can improve the accuracy of the numerical evaluation of the integral. This was not considered here, but it would be expected that a combination of splitting the integral and iterations of the sinh transformation would further improve the accuracy.

\section{References}

[1] Telles, J. C. F. A self-adaptive co-ordinate transformation for efficient numerical evaluation of general boundary element integrals International Journal for Numerical Methods in Engineer- 
ing, 24:959-973, 1987.

[2] Telles, J. C. F. and Oliveira, R. F. Third degree polynomial transformation for boundary element integrals: Further improvements Engineering Analysis with Boundary Elements, 13:135-141, 1994.

[3] Johnston, P. R. Application of sigmoidal transformations to weakly singular and near-singular boundary element integrals International Journal for Numerical Methods in Engineering, 45(10):1333-1348, August 1999.

[4] Zhang, D., Rizzo, F. J. and Rudolphi, Y. J. Stress intensity sensitivities via hypersingular boundary element integral equations Computational Mechanics, 23:389-396, 1999.

[5] Sladek, N., Sladek, J. and Tanaka, M. Regularization of hypersingular and nearly singular integrals in the potential theory and elasticity International Journal for Numerical Methods in Engineering, 36:1609-1628, 1993.

[6] Sladek, V., Sladek, J. and Tanaka, M. Numerical integration of logarithmic and nearly logarithmic singularity in BEMs Applied Mathematical Modelling, 25:901-922, 2001.

[7] Ma, H. and Kamiya, N. Distance transformation for the numerical evaluation of near singular boundary integrals with various kernels in boundary element method Engineering Analysis with Boundary Elements, 26:329-339, 2002.

[8] Johnston, P. R. and Elliott, D. A sinh transformation for evaluating nearly singular boundary element integrals International Journal for Numerical Methods in Engineering, 62(4):564-578, 2005. 
[9] Elliott, D. and Johnston, P. R. Error analysis for a sinh transformation used in evaluating nearly singular boundary element integrals Journal of Computational and Applied Mathematics, 203(1):103-124, 2007.

[10] Spiegel, M. R. Mathematical Handbook of Formulas and Tables Schaum's Outline Series McGraw-Hill, International edition, 1990.

[11] Gradshteyn, I. S. and Ryzhik, I. M. Table of Integrals, Series and Products Academic Press, Boston, $5^{\text {th }}$ edition, 1994. 


\section{Figure and Table Captions}

Figure 1: Effects of zero, one and two iterations of the sinh transformation on the Gauss-Legendre quadrature points with $a_{0}=0.25$ and $b_{0}=0.01$, for 20 integration points. The two horizontal lines indicate how the integration points are clustered around the nearly singular point at $x=0.25$.

Figure 2: Relative errors for the numerical evaluation of the integral $I_{6}$ as a function of distance from the source point to the element, $b$, using various numbers of iterations of the sinh transformation as well as the transformation of Ma and Kamiya [7] with $a=0.2$. Here 40 Gauss-Legendre quadrature points were used in each of the sinh transformation iterations and 20 Gauss-Legendre quadrature points were used in each half of the Ma and Kamiya transformation.

Figure 3: Relative errors for the numerical evaluation of the integral $I_{7}$ as a function of distance from the source point to the element, $b$, using various numbers of iterations of the sinh transformation as well as the transformation of Ma and Kamiya [7] with $a=0.2$. Here 40 Gauss-Legendre quadrature points were used in each of the sinh transformation iterations and 20 Gauss-Legendre quadrature points were used in each half of the Ma and Kamiya transformation.

Table 1: Exact values of the integrals $I_{j}$, for $j=1, \ldots, 5$, for different distances from the source point to the element, $b_{0}$.

Table 2: Relative errors for the evaluation of the integral $I_{1}$ using the Telles and Oliveira self-adaptive transformation [2], the Ma and Kamiya transformation [7] and zero, one and two iterations of the sinh transformation. The integral is evaluated at different distances from the source point to the element, $b_{0}$, and for different numbers of integration points, $n$.

Table 3: Relative errors for the evaluation of the integral $I_{2}$ using the Telles and Oliveira self-adaptive transformation [2], the Ma and Kamiya transformation [7] and zero, one and two iterations of the sinh transformation. The format is the same as that for Table 2 .

Table 4: Relative errors for the evaluation of the integral $I_{3}$ using the Telles and Oliveira self-adaptive 
transformation [2], the Ma and Kamiya transformation [7] and zero, one and two iterations of the sinh transformation. The format is the same as that for Table 2.

Table 5: Relative errors for the evaluation of the integral $I_{4}$ using the Telles and Oliveira self-adaptive transformation [2], the Ma and Kamiya transformation [7] and zero, one and two iterations of the sinh transformation. The format is the same as that for Table 2 .

Table 6: Relative errors for the evaluation of the integral $I_{5}$ using the Ma and Kamiya transformation [7] and zero, one and two iterations of the sinh transformation. The format is the same as that for Table 2. 


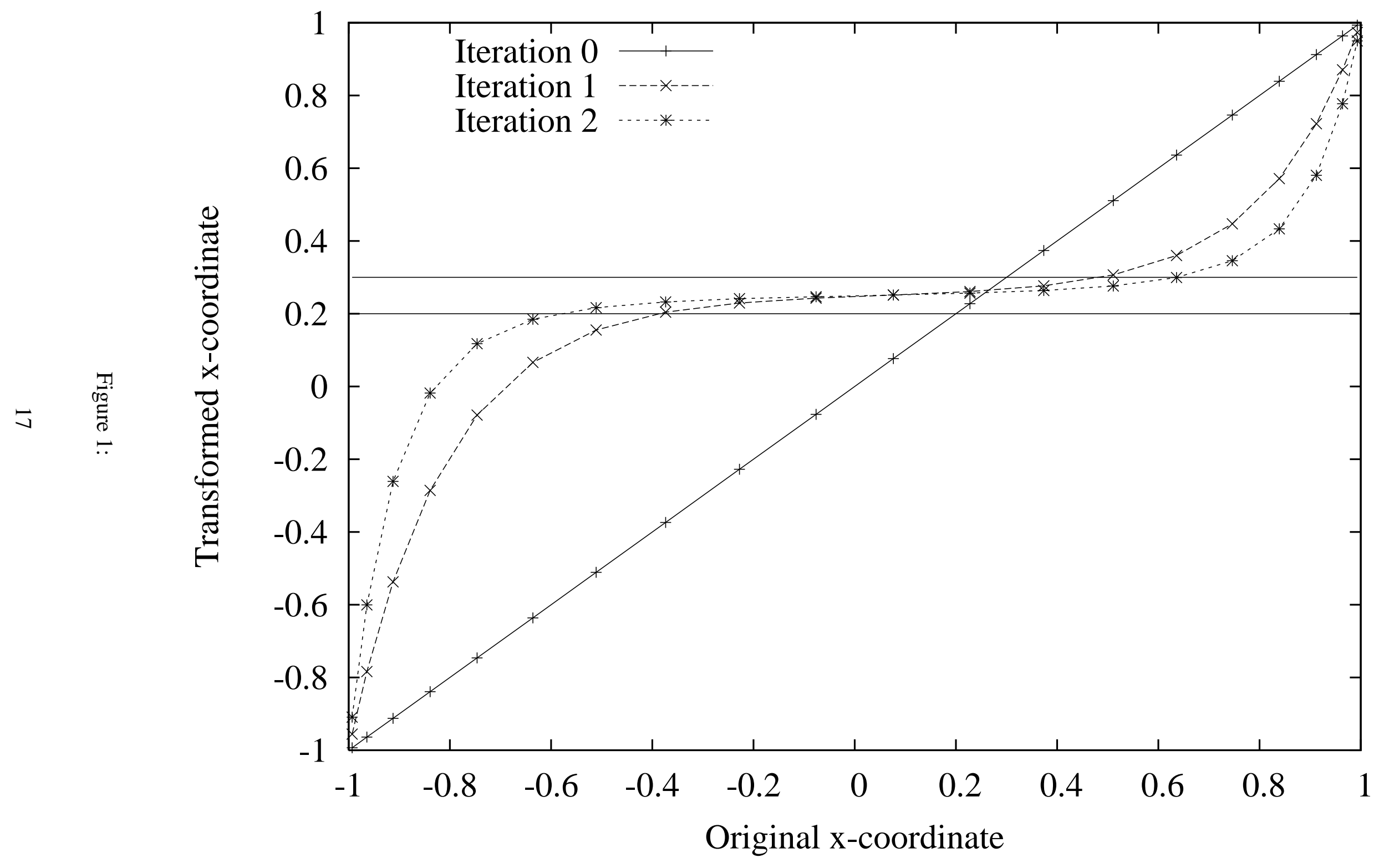









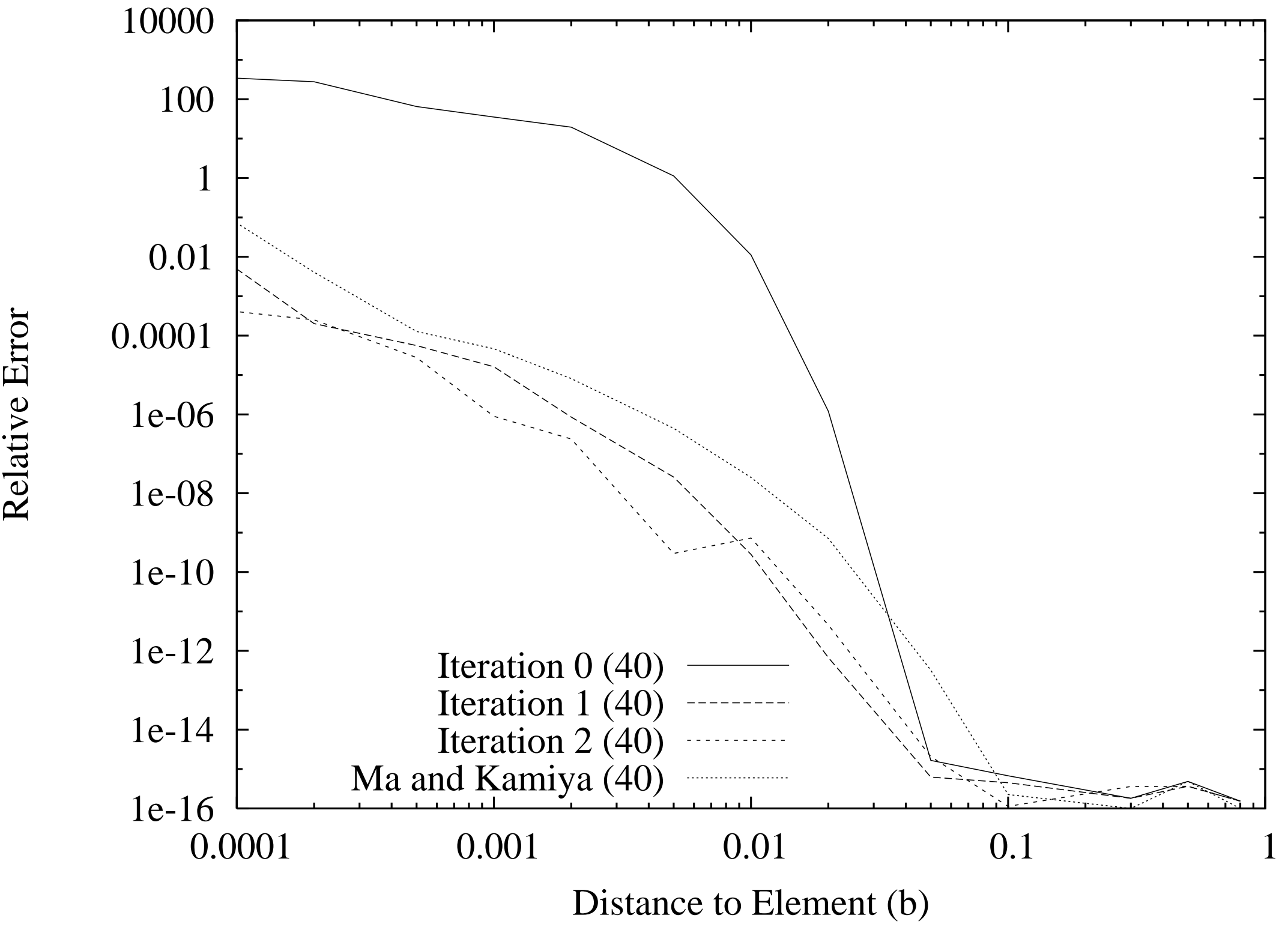




\begin{tabular}{rr|ccccc} 
& $b_{0}$ & $I_{1}$ & $I_{2}$ & $I_{3}$ & $I_{4}$ & \\
& 0.1 & $-1.377280213979331 \times 10^{0}$ & $4.772986570808243 \times 10^{0}$ & $2.600687782291541 \times 10^{1}$ & $1.826962464163693 \times 10^{2}$ & $1.458421664125634 \times 10^{3}$ \\
& 0.01 & $-1.624824690878743 \times 10^{0}$ & $9.061860139411952 \times 10^{0}$ & $2.908109883132889 \times 10^{2}$ & $1.874060113484668 \times 10^{4}$ & $1.472465993683965 \times 10^{6}$ \\
& 0.001 & $-1.651147564185811 \times 10^{0}$ & $1.337869475004172 \times 10^{1}$ & $2.941501665627931 \times 10^{3}$ & $1.874985996065913 \times 10^{6}$ & $1.472619987090925 \times 10^{9}$ \\
& 0.0001 & $-1.653796430462390 \times 10^{0}$ & $1.769603439548428 \times 10^{1}$ & $2.944868685436029 \times 10^{4}$ & $1.874999813908967 \times 10^{8}$ & $1.472621540663769 \times 10^{12}$ \\
0.00001 & $-1.654061483807876 \times 10^{0}$ & $2.201338134801332 \times 10^{1}$ & $2.945205667182708 \times 10^{5}$ & $1.874999997678573 \times 10^{10}$ & $1.472621556213138 \times 10^{15}$ \\
0.000001 & $-1.654087990810535 \times 10^{0}$ & $2.633072839618085 \times 10^{1}$ & $2.945239368156385 \times 10^{6}$ & $1.874999999972181 \times 10^{12}$ & $1.472621556368648 \times 10^{18}$ \\
\hline
\end{tabular}

Table 1: 


\begin{tabular}{|c|c|c|c|c|c|c|}
\hline$b_{0}$ & $n$ & $\begin{array}{l}\text { Telles and } \\
\text { Oliveira[2] }\end{array}$ & $\begin{array}{c}\text { Ma and } \\
\text { Kamiya[7] }\end{array}$ & Iteration 0 & Iteration 1 & Iteration 2 \\
\hline \multirow[t]{5}{*}{0.1} & 10 & $9.7541 \times 10^{-6}$ & $3.2956 \times 10^{-3}$ & $1.0901 \times 10^{-2}$ & $3.2615 \times 10^{-8}$ & $1.8485 \times 10^{-4}$ \\
\hline & 15 & $4.5780 \times 10^{-7}$ & $2.6141 \times 10^{-6}$ & $8.0194 \times 10^{-4}$ & $8.8914 \times 10^{-10}$ & $5.2305 \times 10^{-8}$ \\
\hline & 20 & $2.7726 \times 10^{-10}$ & $8.2077 \times 10^{-8}$ & $1.0714 \times 10^{-3}$ & $5.0884 \times 10^{-12}$ & $3.6081 \times 10^{-12}$ \\
\hline & 25 & $1.9357 \times 10^{-11}$ & $1.3131 \times 10^{-9}$ & $4.2946 \times 10^{-4}$ & $1.7089 \times 10^{-14}$ & $1.6122 \times 10^{-16}$ \\
\hline & 30 & $9.0847 \times 10^{-13}$ & $7.3463 \times 10^{-11}$ & $1.1551 \times 10^{-4}$ & $1.6122 \times 10^{-16}$ & $3.2244 \times 10^{-16}$ \\
\hline \multirow[t]{5}{*}{0.01} & 10 & $7.2515 \times 10^{-4}$ & $1.6406 \times 10^{-2}$ & $7.4987 \times 10^{-2}$ & $5.0077 \times 10^{-5}$ & $1.0180 \times 10^{-2}$ \\
\hline & 15 & $8.7817 \times 10^{-4}$ & $7.0521 \times 10^{-5}$ & $2.0494 \times 10^{-2}$ & $4.0451 \times 10^{-8}$ & $1.0266 \times 10^{-4}$ \\
\hline & 20 & $2.7006 \times 10^{-4}$ & $7.7545 \times 10^{-7}$ & $1.9754 \times 10^{-2}$ & $2.2183 \times 10^{-9}$ & $2.3364 \times 10^{-7}$ \\
\hline & 25 & $2.0660 \times 10^{-6}$ & $5.0644 \times 10^{-9}$ & $5.1374 \times 10^{-2}$ & $1.7689 \times 10^{-10}$ & $2.0456 \times 10^{-10}$ \\
\hline & 30 & $3.9710 \times 10^{-5}$ & $2.1282 \times 10^{-9}$ & $3.8063 \times 10^{-2}$ & $8.8656 \times 10^{-12}$ & $8.8828 \times 10^{-14}$ \\
\hline \multirow[t]{5}{*}{0.001} & 10 & & $3.4543 \times 10^{-2}$ & $8.8599 \times 10^{-2}$ & $1.1121 \times 10^{-3}$ & $3.3658 \times 10^{-2}$ \\
\hline & 15 & & $7.2079 \times 10^{-4}$ & $3.3347 \times 10^{-2}$ & $5.5480 \times 10^{-7}$ & $2.6674 \times 10^{-3}$ \\
\hline & 20 & & $1.3380 \times 10^{-5}$ & $1.1849 \times 10^{-2}$ & $1.2093 \times 10^{-8}$ & $3.9696 \times 10^{-5}$ \\
\hline & 25 & & $1.3571 \times 10^{-8}$ & $7.8515 \times 10^{-2}$ & $4.3573 \times 10^{-10}$ & $2.2625 \times 10^{-7}$ \\
\hline & 30 & & $5.0562 \times 10^{-10}$ & $6.9897 \times 10^{-2}$ & $1.6341 \times 10^{-10}$ & $6.3635 \times 10^{-10}$ \\
\hline \multirow[t]{5}{*}{0.0001} & 10 & & $4.3489 \times 10^{-2}$ & $9.0047 \times 10^{-2}$ & $5.6818 \times 10^{-3}$ & $2.0285 \times 10^{-2}$ \\
\hline & 15 & & $3.0377 \times 10^{-3}$ & $3.4868 \times 10^{-2}$ & $6.8412 \times 10^{-6}$ & $1.3398 \times 10^{-2}$ \\
\hline & 20 & & $1.2173 \times 10^{-4}$ & $1.0319 \times 10^{-2}$ & $3.0321 \times 10^{-8}$ & $6.8031 \times 10^{-4}$ \\
\hline & 25 & & $2.6266 \times 10^{-7}$ & $7.7676 \times 10^{-2}$ & $2.2324 \times 10^{-9}$ & $1.3165 \times 10^{-5}$ \\
\hline & 30 & & $6.1648 \times 10^{-10}$ & $6.9458 \times 10^{-2}$ & $2.4556 \times 10^{-11}$ & $1.2695 \times 10^{-7}$ \\
\hline \multirow[t]{5}{*}{0.00001} & 10 & & $3.4423 \times 10^{-2}$ & $9.0196 \times 10^{-2}$ & $1.4792 \times 10^{-2}$ & $5.6625 \times 10^{-2}$ \\
\hline & 15 & & $7.8961 \times 10^{-3}$ & $3.5022 \times 10^{-2}$ & $6.8335 \times 10^{-5}$ & $3.0057 \times 10^{-2}$ \\
\hline & 20 & & $5.5849 \times 10^{-4}$ & $1.0159 \times 10^{-2}$ & $3.8002 \times 10^{-9}$ & $3.7642 \times 10^{-3}$ \\
\hline & 25 & & $2.8437 \times 10^{-6}$ & $7.7512 \times 10^{-2}$ & $2.7269 \times 10^{-9}$ & $1.7440 \times 10^{-4}$ \\
\hline & 30 & & $4.0656 \times 10^{-8}$ & $6.9302 \times 10^{-2}$ & $3.2817 \times 10^{-10}$ & $4.0813 \times 10^{-6}$ \\
\hline \multirow[t]{5}{*}{0.000001} & 10 & & $5.2536 \times 10^{-3}$ & $9.0207 \times 10^{-2}$ & $2.6561 \times 10^{-2}$ & $1.7803 \times 10^{-1}$ \\
\hline & 15 & & $1.5154 \times 10^{-2}$ & $3.5037 \times 10^{-2}$ & $3.4808 \times 10^{-4}$ & $4.1221 \times 10^{-2}$ \\
\hline & 20 & & $1.6824 \times 10^{-3}$ & $1.0142 \times 10^{-2}$ & $3.9536 \times 10^{-7}$ & $1.1036 \times 10^{-2}$ \\
\hline & 25 & & $1.7525 \times 10^{-5}$ & $7.7499 \times 10^{-2}$ & $1.4228 \times 10^{-9}$ & $9.8925 \times 10^{-4}$ \\
\hline & 30 & & $4.0501 \times 10^{-7}$ & $6.9283 \times 10^{-2}$ & $2.5204 \times 10^{-10}$ & $4.5316 \times 10^{-5}$ \\
\hline
\end{tabular}

Table 2: 


\begin{tabular}{|c|c|c|c|c|c|c|}
\hline$b_{0}$ & $n$ & $\begin{array}{l}\text { Telles and } \\
\text { Oliveira[2] }\end{array}$ & $\begin{array}{c}\text { Ma and } \\
\text { Kamiya[7] }\end{array}$ & Iteration 0 & Iteration 1 & Iteration 2 \\
\hline \multirow[t]{5}{*}{0.1} & 10 & $6.1011 \times 10^{-5}$ & $6.1774 \times 10^{-4}$ & $3.7637 \times 10^{-2}$ & $1.8346 \times 10^{-11}$ & $2.2359 \times 10^{-6}$ \\
\hline & 15 & $6.8701 \times 10^{-7}$ & $8.5247 \times 10^{-6}$ & $2.3361 \times 10^{-3}$ & $3.7218 \times 10^{-16}$ & $8.9212 \times 10^{-11}$ \\
\hline & 20 & $7.5213 \times 10^{-9}$ & $4.7085 \times 10^{-7}$ & $4.7742 \times 10^{-3}$ & $1.8608 \times 10^{-16}$ & $1.1165 \times 10^{-15}$ \\
\hline & 25 & $1.2932 \times 10^{-10}$ & $4.1281 \times 10^{-9}$ & $2.1978 \times 10^{-3}$ & $0.0000 \times 10^{+00}$ & $1.8608 \times 10^{-16}$ \\
\hline & 30 & $9.1405 \times 10^{-13}$ & $3.0609 \times 10^{-11}$ & $6.5785 \times 10^{-4}$ & $1.8608 \times 10^{-16}$ & $1.8608 \times 10^{-16}$ \\
\hline \multirow[t]{5}{*}{0.01} & 10 & $4.5881 \times 10^{-3}$ & $2.2767 \times 10^{-3}$ & $3.8395 \times 10^{-1}$ & $3.8039 \times 10^{-8}$ & $2.9066 \times 10^{-4}$ \\
\hline & 15 & $2.9275 \times 10^{-3}$ & $2.9661 \times 10^{-5}$ & $2.0792 \times 10^{-1}$ & $3.5284 \times 10^{-15}$ & $3.6078 \times 10^{-7}$ \\
\hline & 20 & $3.3319 \times 10^{-4}$ & $1.5771 \times 10^{-5}$ & $6.1187 \times 10^{-2}$ & $1.9603 \times 10^{-16}$ & $1.4686 \times 10^{-10}$ \\
\hline & 25 & $5.7075 \times 10^{-6}$ & $4.7182 \times 10^{-7}$ & $5.1925 \times 10^{-1}$ & $3.9205 \times 10^{-16}$ & $2.7640 \times 10^{-14}$ \\
\hline & 30 & $6.6224 \times 10^{-6}$ & $8.2593 \times 10^{-8}$ & $4.3270 \times 10^{-1}$ & $1.9603 \times 10^{-16}$ & $3.9205 \times 10^{-16}$ \\
\hline \multirow[t]{5}{*}{0.001} & 10 & & $1.2572 \times 10^{-3}$ & $5.8154 \times 10^{-1}$ & $1.8163 \times 10^{-6}$ & $2.2667 \times 10^{-3}$ \\
\hline & 15 & & $1.2005 \times 10^{-5}$ & $4.5735 \times 10^{-1}$ & $5.0103 \times 10^{-12}$ & $1.7418 \times 10^{-5}$ \\
\hline & 20 & & $6.6160 \times 10^{-5}$ & $2.3937 \times 10^{-1}$ & $1.3278 \times 10^{-15}$ & $4.5133 \times 10^{-8}$ \\
\hline & 25 & & $5.3701 \times 10^{-6}$ & $6.6515 \times 10^{-1}$ & $1.3278 \times 10^{-16}$ & $5.3712 \times 10^{-11}$ \\
\hline & 30 & & $1.0856 \times 10^{-6}$ & $8.0725 \times 10^{-1}$ & $6.6388 \times 10^{-16}$ & $3.5850 \times 10^{-14}$ \\
\hline \multirow[t]{5}{*}{0.0001} & 10 & & $1.0352 \times 10^{-2}$ & $6.8360 \times 10^{-1}$ & $1.8068 \times 10^{-5}$ & $6.3398 \times 10^{-3}$ \\
\hline & 15 & & $7.7421 \times 10^{-4}$ & $5.8968 \times 10^{-1}$ & $4.6118 \times 10^{-10}$ & $1.5597 \times 10^{-4}$ \\
\hline & 20 & & $1.9258 \times 10^{-4}$ & $4.2458 \times 10^{-1}$ & $9.2348 \times 10^{-15}$ & $1.3522 \times 10^{-6}$ \\
\hline & 25 & & $1.8807 \times 10^{-5}$ & $2.7226 \times 10^{-1}$ & $4.4168 \times 10^{-15}$ & $5.4925 \times 10^{-9}$ \\
\hline & 30 & & $5.2761 \times 10^{-6}$ & $3.9159 \times 10^{-1}$ & $7.4282 \times 10^{-15}$ & $1.2327 \times 10^{-11}$ \\
\hline \multirow[t]{5}{*}{0.00001} & 10 & & $1.2214 \times 10^{-2}$ & $7.4568 \times 10^{-1}$ & $8.0392 \times 10^{-5}$ & $1.0995 \times 10^{-2}$ \\
\hline & 15 & & $3.5478 \times 10^{-4}$ & $6.7018 \times 10^{-1}$ & $1.0336 \times 10^{-8}$ & $6.0799 \times 10^{-4}$ \\
\hline & 20 & & $2.2635 \times 10^{-5}$ & $5.3745 \times 10^{-1}$ & $1.5025 \times 10^{-13}$ & $1.2472 \times 10^{-5}$ \\
\hline & 25 & & $4.8423 \times 10^{-5}$ & $2.2850 \times 10^{-2}$ & $6.9399 \times 10^{-14}$ & $1.2325 \times 10^{-7}$ \\
\hline & 30 & & $1.2031 \times 10^{-5}$ & $1.1888 \times 10^{-1}$ & $2.8405 \times 10^{-14}$ & $6.8290 \times 10^{-10}$ \\
\hline \multirow[t]{5}{*}{0.000001} & 10 & & $8.4724 \times 10^{-3}$ & $7.8737 \times 10^{-1}$ & $2.2492 \times 10^{-4}$ & $1.4802 \times 10^{-2}$ \\
\hline & 15 & & $1.7007 \times 10^{-3}$ & $7.2425 \times 10^{-1}$ & $9.8501 \times 10^{-8}$ & $1.4835 \times 10^{-3}$ \\
\hline & 20 & & $5.4050 \times 10^{-4}$ & $6.1328 \times 10^{-1}$ & $2.9194 \times 10^{-12}$ & $5.8035 \times 10^{-5}$ \\
\hline & 25 & & $9.2354 \times 10^{-6}$ & $1.4486 \times 10^{-1}$ & $5.3591 \times 10^{-13}$ & $1.1300 \times 10^{-6}$ \\
\hline & 30 & & $2.2429 \times 10^{-6}$ & $6.4579 \times 10^{-2}$ & $2.5582 \times 10^{-13}$ & $1.2571 \times 10^{-8}$ \\
\hline
\end{tabular}

Table 3: 


\begin{tabular}{|c|c|c|c|c|c|c|}
\hline$b_{0}$ & $n$ & $\begin{array}{l}\text { Telles and } \\
\text { Oliveira[2] }\end{array}$ & $\begin{array}{c}\text { Ma and } \\
\text { Kamiya[7] }\end{array}$ & Iteration 0 & Iteration 1 & Iteration 2 \\
\hline \multirow[t]{5}{*}{0.1} & 10 & $9.1284 \times 10^{-4}$ & $2.3659 \times 10^{-3}$ & $1.4086 \times 10^{-1}$ & $3.3754 \times 10^{-6}$ & $4.1270 \times 10^{-9}$ \\
\hline & 15 & $6.0822 \times 10^{-6}$ & $2.6471 \times 10^{-5}$ & $6.6559 \times 10^{-3}$ & $4.2570 \times 10^{-7}$ & $3.9451 \times 10^{-13}$ \\
\hline & 20 & $1.5644 \times 10^{-6}$ & $6.8471 \times 10^{-7}$ & $2.2522 \times 10^{-2}$ & $3.9566 \times 10^{-9}$ & $0.0000 \times 10^{+00}$ \\
\hline & 25 & $7.8967 \times 10^{-9}$ & $2.7174 \times 10^{-8}$ & $1.1760 \times 10^{-2}$ & $1.9286 \times 10^{-11}$ & $4.0981 \times 10^{-16}$ \\
\hline & 30 & $1.8558 \times 10^{-9}$ & $3.8993 \times 10^{-9}$ & $3.8824 \times 10^{-3}$ & $2.2404 \times 10^{-14}$ & $1.3661 \times 10^{-16}$ \\
\hline \multirow[t]{5}{*}{0.01} & 10 & $1.2143 \times 10^{-3}$ & $1.5566 \times 10^{-2}$ & $8.7050 \times 10^{-1}$ & $4.6976 \times 10^{-3}$ & $8.6692 \times 10^{-7}$ \\
\hline & 15 & $1.5905 \times 10^{-2}$ & $9.7852 \times 10^{-4}$ & $6.8866 \times 10^{-1}$ & $5.8798 \times 10^{-5}$ & $6.5672 \times 10^{-10}$ \\
\hline & 20 & $4.0328 \times 10^{-3}$ & $8.0676 \times 10^{-5}$ & $1.1916 \times 10^{-1}$ & $7.5193 \times 10^{-6}$ & $3.0414 \times 10^{-13}$ \\
\hline & 25 & $1.6650 \times 10^{-4}$ & $8.0485 \times 10^{-6}$ & $1.8837 \times 10^{+0}$ & $8.7249 \times 10^{-7}$ & $5.8639 \times 10^{-16}$ \\
\hline & 30 & $3.0927 \times 10^{-6}$ & $1.1947 \times 10^{-7}$ & $1.7297 \times 10^{+0}$ & $6.1325 \times 10^{-8}$ & $1.9546 \times 10^{-16}$ \\
\hline \multirow[t]{5}{*}{0.001} & 10 & & $4.4340 \times 10^{-2}$ & $9.8708 \times 10^{-1}$ & $4.0194 \times 10^{-2}$ & $8.7829 \times 10^{-6}$ \\
\hline & 15 & & $4.0768 \times 10^{-3}$ & $9.6811 \times 10^{-1}$ & $3.4693 \times 10^{-3}$ & $1.5891 \times 10^{-8}$ \\
\hline & 20 & & $6.1359 \times 10^{-5}$ & $8.9668 \times 10^{-1}$ & $1.8263 \times 10^{-4}$ & $2.2940 \times 10^{-11}$ \\
\hline & 25 & & $5.9407 \times 10^{-6}$ & $3.2903 \times 10^{-3}$ & $1.2371 \times 10^{-5}$ & $3.5094 \times 10^{-14}$ \\
\hline & 30 & & $1.6861 \times 10^{-5}$ & $4.1652 \times 10^{-1}$ & $5.9330 \times 10^{-6}$ & $4.1741 \times 10^{-15}$ \\
\hline \multirow[t]{5}{*}{0.0001} & 10 & & $6.7915 \times 10^{-2}$ & $9.9872 \times 10^{-1}$ & $1.2006 \times 10^{-1}$ & $3.0635 \times 10^{-5}$ \\
\hline & 15 & & $1.7393 \times 10^{-3}$ & $9.9682 \times 10^{-1}$ & $2.0564 \times 10^{-2}$ & $9.9393 \times 10^{-8}$ \\
\hline & 20 & & $2.0854 \times 10^{-3}$ & $9.8965 \times 10^{-1}$ & $3.0042 \times 10^{-3}$ & $2.8017 \times 10^{-10}$ \\
\hline & 25 & & $2.0573 \times 10^{-5}$ & $8.9783 \times 10^{-1}$ & $3.2494 \times 10^{-4}$ & $8.0360 \times 10^{-13}$ \\
\hline & 30 & & $6.0341 \times 10^{-5}$ & $8.5206 \times 10^{-1}$ & $2.9834 \times 10^{-6}$ & $4.2002 \times 10^{-15}$ \\
\hline \multirow[t]{5}{*}{0.00001} & 10 & & $2.3184 \times 10^{-2}$ & $9.9986 \times 10^{-1}$ & $2.2969 \times 10^{-1}$ & $7.2012 \times 10^{-5}$ \\
\hline & 15 & & $2.1627 \times 10^{-2}$ & $9.9969 \times 10^{-1}$ & $5.9347 \times 10^{-2}$ & $3.5441 \times 10^{-7}$ \\
\hline & 20 & & $5.8159 \times 10^{-3}$ & $9.9898 \times 10^{-1}$ & $1.3426 \times 10^{-2}$ & $1.5373 \times 10^{-9}$ \\
\hline & 25 & & $8.3287 \times 10^{-4}$ & $9.8978 \times 10^{-1}$ & $2.7432 \times 10^{-3}$ & $6.1096 \times 10^{-12}$ \\
\hline & 30 & & $2.8763 \times 10^{-4}$ & $9.8519 \times 10^{-1}$ & $4.6221 \times 10^{-4}$ & $1.5574 \times 10^{-13}$ \\
\hline \multirow[t]{5}{*}{0.000001} & 10 & & $1.4089 \times 10^{-1}$ & $9.9999 \times 10^{-1}$ & $3.4904 \times 10^{-1}$ & $1.4501 \times 10^{-4}$ \\
\hline & 15 & & $2.3148 \times 10^{-2}$ & $9.9995 \times 10^{-1}$ & $1.2101 \times 10^{-1}$ & $9.3782 \times 10^{-7}$ \\
\hline & 20 & & $7.5537 \times 10^{-3}$ & $9.9988 \times 10^{-1}$ & $3.5335 \times 10^{-2}$ & $5.3646 \times 10^{-9}$ \\
\hline & 25 & & $1.7200 \times 10^{-3}$ & $9.9897 \times 10^{-1}$ & $9.9618 \times 10^{-3}$ & $3.1284 \times 10^{-11}$ \\
\hline & 30 & & $5.7267 \times 10^{-4}$ & $9.9853 \times 10^{-1}$ & $2.5951 \times 10^{-3}$ & $1.6732 \times 10^{-12}$ \\
\hline
\end{tabular}

Table 4: 


\begin{tabular}{|c|c|c|c|c|c|c|}
\hline$b_{0}$ & $n$ & $\begin{array}{l}\text { Telles and } \\
\text { Oliveira[2] }\end{array}$ & $\begin{array}{c}\text { Ma and } \\
\text { Kamiya[7] }\end{array}$ & Iteration 0 & Iteration 1 & Iteration 2 \\
\hline \multirow[t]{5}{*}{0.1} & 10 & $3.6570 \times 10^{-3}$ & $1.8251 \times 10^{-3}$ & $2.8305 \times 10^{-1}$ & $2.8390 \times 10^{-6}$ & $8.5990 \times 10^{-7}$ \\
\hline & 15 & $1.7296 \times 10^{-4}$ & $2.6340 \times 10^{-5}$ & $8.0812 \times 10^{-3}$ & $5.8299 \times 10^{-6}$ & $6.7889 \times 10^{-11}$ \\
\hline & 20 & $3.2072 \times 10^{-6}$ & $5.8419 \times 10^{-6}$ & $5.5546 \times 10^{-2}$ & $6.9098 \times 10^{-8}$ & $1.2445 \times 10^{-15}$ \\
\hline & 25 & $2.6163 \times 10^{-7}$ & $2.2976 \times 10^{-7}$ & $3.2514 \times 10^{-2}$ & $4.0589 \times 10^{-10}$ & $0.0000 \times 10^{0}$ \\
\hline & 30 & $7.4175 \times 10^{-9}$ & $2.0877 \times 10^{-8}$ & $1.1735 \times 10^{-2}$ & $4.3792 \times 10^{-13}$ & $7.7785 \times 10^{-16}$ \\
\hline \multirow[t]{5}{*}{0.01} & 10 & $4.4246 \times 10^{-2}$ & $5.9512 \times 10^{-3}$ & $9.8305 \times 10^{-1}$ & $2.7404 \times 10^{-2}$ & $8.4288 \times 10^{-5}$ \\
\hline & 15 & $8.4775 \times 10^{-3}$ & $2.6360 \times 10^{-3}$ & $9.1400 \times 10^{-1}$ & $4.6410 \times 10^{-4}$ & $9.4928 \times 10^{-8}$ \\
\hline & 20 & $9.0341 \times 10^{-3}$ & $1.1604 \times 10^{-4}$ & $4.7231 \times 10^{-1}$ & $9.0531 \times 10^{-5}$ & $8.1737 \times 10^{-11}$ \\
\hline & 25 & $5.9159 \times 10^{-4}$ & $1.8273 \times 10^{-5}$ & $2.7217 \times 10^{0}$ & $1.2741 \times 10^{-5}$ & $5.8627 \times 10^{-14}$ \\
\hline & 30 & $2.2988 \times 10^{-4}$ & $2.7472 \times 10^{-6}$ & $2.7225 \times 10^{0}$ & $1.0619 \times 10^{-6}$ & $1.9412 \times 10^{-16}$ \\
\hline \multirow[t]{5}{*}{0.001} & 10 & & $1.0051 \times 10^{-1}$ & $9.9985 \times 10^{-1}$ & $1.7019 \times 10^{-1}$ & $5.9760 \times 10^{-4}$ \\
\hline & 15 & & $1.1675 \times 10^{-2}$ & $9.9910 \times 10^{-1}$ & $2.1573 \times 10^{-2}$ & $1.9689 \times 10^{-6}$ \\
\hline & 20 & & $1.9450 \times 10^{-3}$ & $9.9307 \times 10^{-1}$ & $1.4756 \times 10^{-3}$ & $5.1425 \times 10^{-9}$ \\
\hline & 25 & & $2.0601 \times 10^{-4}$ & $7.5019 \times 10^{-1}$ & $1.3566 \times 10^{-4}$ & $1.1676 \times 10^{-11}$ \\
\hline & 30 & & $1.5771 \times 10^{-5}$ & $5.3451 \times 10^{-1}$ & $7.4534 \times 10^{-5}$ & $1.9744 \times 10^{-14}$ \\
\hline \multirow[t]{5}{*}{0.0001} & 10 & & $9.9552 \times 10^{-4}$ & $1.0000 \times 10^{0}$ & $3.8919 \times 10^{-1}$ & $1.8185 \times 10^{-3}$ \\
\hline & 15 & & $1.7910 \times 10^{-2}$ & $1.0000 \times 10^{0}$ & $9.9824 \times 10^{-2}$ & $1.1098 \times 10^{-5}$ \\
\hline & 20 & & $8.8904 \times 10^{-4}$ & $9.9995 \times 10^{-1}$ & $1.9260 \times 10^{-2}$ & $5.4139 \times 10^{-8}$ \\
\hline & 25 & & $8.4743 \times 10^{-4}$ & $9.9739 \times 10^{-1}$ & $2.5643 \times 10^{-3}$ & $2.3193 \times 10^{-10}$ \\
\hline & 30 & & $7.2949 \times 10^{-5}$ & $9.9504 \times 10^{-1}$ & $1.9033 \times 10^{-5}$ & $8.9472 \times 10^{-13}$ \\
\hline \multirow[t]{5}{*}{0.00001} & 10 & & $1.9436 \times 10^{-1}$ & $1.0000 \times 10^{0}$ & $5.9509 \times 10^{-1}$ & $3.7773 \times 10^{-3}$ \\
\hline & 15 & & $4.0433 \times 10^{-2}$ & $1.0000 \times 10^{0}$ & $2.3672 \times 10^{-1}$ & $3.4867 \times 10^{-5}$ \\
\hline & 20 & & $1.5302 \times 10^{-2}$ & $1.0000 \times 10^{0}$ & $7.0341 \times 10^{-2}$ & $2.5780 \times 10^{-7}$ \\
\hline & 25 & & $5.8981 \times 10^{-5}$ & $1.0000 \times 10^{0}$ & $1.7797 \times 10^{-2}$ & $1.6776 \times 10^{-9}$ \\
\hline & 30 & & $2.4516 \times 10^{-4}$ & $9.9995 \times 10^{-1}$ & $3.5821 \times 10^{-3}$ & $9.6917 \times 10^{-12}$ \\
\hline \multirow[t]{5}{*}{0.000001} & 10 & & $2.8434 \times 10^{-1}$ & $1.0000 \times 10^{0}$ & $7.4891 \times 10^{-1}$ & $6.3861 \times 10^{-3}$ \\
\hline & 15 & & $8.9785 \times 10^{-3}$ & $1.0000 \times 10^{0}$ & $4.1293 \times 10^{-1}$ & $7.9845 \times 10^{-5}$ \\
\hline & 20 & & $4.3260 \times 10^{-3}$ & $1.0000 \times 10^{0}$ & $1.5579 \times 10^{-1}$ & $7.9904 \times 10^{-7}$ \\
\hline & 25 & & $5.7658 \times 10^{-3}$ & $1.0000 \times 10^{0}$ & $5.4619 \times 10^{-2}$ & $7.0448 \times 10^{-9}$ \\
\hline & 30 & & $2.0352 \times 10^{-3}$ & $1.0000 \times 10^{0}$ & $1.7049 \times 10^{-2}$ & $6.0768 \times 10^{-11}$ \\
\hline
\end{tabular}

Table 5: 


\begin{tabular}{|c|c|c|c|c|c|}
\hline$b_{0}$ & $n$ & Ma and Kamiya[7] & Iteration 0 & Iteration 1 & Iteration 2 \\
\hline \multirow[t]{5}{*}{0.1} & 10 & $3.3393 \times 10^{-3}$ & $4.2689 \times 10^{-1}$ & $1.3534 \times 10^{-4}$ & $1.3009 \times 10^{-5}$ \\
\hline & 15 & $2.1193 \times 10^{-4}$ & $2.4647 \times 10^{-3}$ & $3.5894 \times 10^{-5}$ & $1.9830 \times 10^{-9}$ \\
\hline & 20 & $2.2729 \times 10^{-5}$ & $1.0012 \times 10^{-1}$ & $5.3986 \times 10^{-7}$ & $3.3519 \times 10^{-14}$ \\
\hline & 25 & $5.7890 \times 10^{-7}$ & $6.4980 \times 10^{-2}$ & $3.8052 \times 10^{-9}$ & $4.6771 \times 10^{-16}$ \\
\hline & 30 & $4.0351 \times 10^{-8}$ & $2.5427 \times 10^{-2}$ & $3.4854 \times 10^{-12}$ & $3.1181 \times 10^{-16}$ \\
\hline \multirow[t]{5}{*}{0.01} & 10 & $2.8079 \times 10^{-2}$ & $9.9799 \times 10^{-1}$ & $7.1323 \times 10^{-2}$ & $9.4067 \times 10^{-4}$ \\
\hline & 15 & $2.2557 \times 10^{-3}$ & $9.7870 \times 10^{-1}$ & $1.5706 \times 10^{-3}$ & $2.1828 \times 10^{-6}$ \\
\hline & 20 & $7.4214 \times 10^{-4}$ & $7.2681 \times 10^{-1}$ & $4.5570 \times 10^{-4}$ & $3.1804 \times 10^{-9}$ \\
\hline & 25 & $1.6764 \times 10^{-6}$ & $3.0298 \times 10^{0}$ & $7.7516 \times 10^{-5}$ & $3.4008 \times 10^{-12}$ \\
\hline & 30 & $9.7214 \times 10^{-6}$ & $3.2860 \times 10^{0}$ & $7.6328 \times 10^{-6}$ & $2.5300 \times 10^{-15}$ \\
\hline \multirow[t]{5}{*}{0.001} & 10 & $9.3078 \times 10^{-2}$ & $9.9999 \times 10^{-1}$ & $3.3447 \times 10^{-1}$ & $5.3456 \times 10^{-3}$ \\
\hline & 15 & $1.1270 \times 10^{-2}$ & $9.9999 \times 10^{-1}$ & $5.8250 \times 10^{-2}$ & $3.6574 \times 10^{-5}$ \\
\hline & 20 & $4.8958 \times 10^{-3}$ & $9.9958 \times 10^{-1}$ & $5.0624 \times 10^{-3}$ & $1.6288 \times 10^{-7}$ \\
\hline & 25 & $5.8331 \times 10^{-4}$ & $9.4885 \times 10^{-1}$ & $6.1383 \times 10^{-4}$ & $5.6285 \times 10^{-10}$ \\
\hline & 30 & $6.9074 \times 10^{-5}$ & $8.7511 \times 10^{-1}$ & $3.8666 \times 10^{-4}$ & $1.6310 \times 10^{-12}$ \\
\hline \multirow[t]{5}{*}{0.0001} & 10 & $1.2071 \times 10^{-1}$ & $9.9999 \times 10^{-1}$ & $6.2146 \times 10^{-1}$ & $1.3933 \times 10^{-2}$ \\
\hline & 15 & $4.1020 \times 10^{-2}$ & $9.9999 \times 10^{-1}$ & $2.1796 \times 10^{-1}$ & $1.7675 \times 10^{-4}$ \\
\hline & 20 & $9.7563 \times 10^{-3}$ & $9.9999 \times 10^{-1}$ & $5.3374 \times 10^{-2}$ & $1.4724 \times 10^{-6}$ \\
\hline & 25 & $2.1840 \times 10^{-3}$ & $9.9992 \times 10^{-1}$ & $8.5575 \times 10^{-3}$ & $9.6162 \times 10^{-9}$ \\
\hline & 30 & $5.4143 \times 10^{-4}$ & $9.9985 \times 10^{-1}$ & $4.4257 \times 10^{-5}$ & $5.3450 \times 10^{-11}$ \\
\hline \multirow[t]{5}{*}{0.00001} & 10 & $2.7789 \times 10^{-1}$ & $9.9999 \times 10^{-1}$ & $8.1406 \times 10^{-1}$ & $2.5823 \times 10^{-2}$ \\
\hline & 15 & $2.1870 \times 10^{-2}$ & $9.9999 \times 10^{-1}$ & $4.4521 \times 10^{-1}$ & $4.9425 \times 10^{-4}$ \\
\hline & 20 & $1.2612 \times 10^{-2}$ & $9.9999 \times 10^{-1}$ & $1.6346 \times 10^{-1}$ & $6.2366 \times 10^{-6}$ \\
\hline & 25 & $3.5280 \times 10^{-3}$ & $9.9999 \times 10^{-1}$ & $4.9615 \times 10^{-2}$ & $6.1922 \times 10^{-8}$ \\
\hline & 30 & $8.7575 \times 10^{-4}$ & $9.9999 \times 10^{-1}$ & $1.1762 \times 10^{-2}$ & $5.2512 \times 10^{-10}$ \\
\hline \multirow[t]{5}{*}{0.000001} & 10 & $2.6287 \times 10^{-1}$ & $9.9999 \times 10^{-1}$ & $9.1633 \times 10^{-1}$ & $3.9917 \times 10^{-2}$ \\
\hline & 15 & $6.8564 \times 10^{-2}$ & $9.9999 \times 10^{-1}$ & $7.0072 \times 10^{-1}$ & $1.0305 \times 10^{-3}$ \\
\hline & 20 & $3.0842 \times 10^{-2}$ & $9.9999 \times 10^{-1}$ & $3.1372 \times 10^{-1}$ & $1.7582 \times 10^{-5}$ \\
\hline & 25 & $5.8948 \times 10^{-3}$ & $9.9999 \times 10^{-1}$ & $1.3143 \times 10^{-1}$ & $2.3645 \times 10^{-7}$ \\
\hline & 30 & $2.1127 \times 10^{-3}$ & $9.9999 \times 10^{-1}$ & $4.8158 \times 10^{-2}$ & $2.7290 \times 10^{-9}$ \\
\hline
\end{tabular}

Table 6: 